\title{
Evaluation of outbreak response immunization in the control of pertussis using agent-based modeling
}

\author{
Alexander Doroshenko ${ }^{\text {Corresp., }}{ }^{2}$, Weicheng Qian ${ }^{2}$, Nathaniel D Osgood ${ }^{2}$ \\ ${ }^{1}$ Faculty of Medicine and Dentistry, Department of Medicine, Division of Preventive Medicine, University of Alberta, Edmonton, Alberta, Canada \\ 2 Department of Computer Science, University of Saskatchewan, Saskatoon, Saskatchewan, Canada \\ Corresponding Author: Alexander Doroshenko \\ Email address: adoroshe@ualberta.ca
}

Background. Pertussis control remains a challenge due to recently observed effects of waning immunity to acellular vaccine and suboptimal vaccine coverage. Multiple outbreaks have been reported in different ages worldwide. For certain outbreaks, public health authorities can launch an outbreak response immunization campaign to control pertussis spread. We investigated effects of an outbreak response immunization targeting young adolescents in averting pertussis cases. Methods. We developed an agent-based model for pertussis transmission representing disease mechanism, waning immunity, vaccination schedule and pathogen transmission in a spatially-explicit 500,000-person contact network representing a typical Canadian Public Health district. Parameters were derived from literature and calibration. We used published cumulative incidence and dose-specific vaccine coverage to calibrate the model's epidemiological curves. We endogenized outbreak response by defining thresholds to trigger simulated immunization campaigns in the 10-14 age group offering $80 \%$ coverage. We ran paired simulations with and without outbreak response immunization and included those resulting in a single ORI within a 10year span. We calculated the number of cases averted attributable to outbreak immunization campaign in all ages, in the 10-14 age group and in infants. The count of cases averted were tested using Mann-Whitney $U$ test to determine statistical significance. Numbers needed to vaccinate during immunization campaign to prevent a single case in respective age groups were derived from the model. We varied adult vaccine coverage, waning immunity parameters, immunization campaign eligibility and tested stronger vaccination boosting effect in sensitivity analyses. Results. 189 qualified paired-runs were analyzed. On average, ORI was triggered every 26 years. On a per-run basis, there were an average of 124, 243 and 429 pertussis cases averted across all age groups within 1, 3 and 10 years of a campaign, respectively. During the same time periods, 53, 96, and 163 cases were averted in the 10-14 age group, and 6, 11, 20 in infants under 1 ( $p<0.001$, all groups). Numbers needed to vaccinate ranged from 49 to 221, from 130 to 519 and from 
1031 to 4903 for all ages, the 10-14 age group and for infants, respectively. Most sensitivity analyses resulted in minimal impact on a number of cases averted. Discussion. Our model generated 30 years of longitudinal data to evaluate effects of outbreak response immunization in a controlled study. Immunization campaign implemented as an outbreak response measure among adolescents may confer benefits across all ages accruing over a 10-year period. Our inference is dependent on having an outbreak of significant magnitude affecting predominantly the selected age and achieving a comprehensive vaccine coverage during the campaign. Economic evaluations and comparisons with other control measures can add to conclusions generated by our work. 
1 Evaluation of outbreak response immunization in the control of pertussis using agent-

2 based modeling

3 Alexander Doroshenko ${ }^{1}$, Weicheng Qian ${ }^{2}$, Nathaniel D Osgood ${ }^{2}$

$4{ }^{1}$ Department of Medicine, University of Alberta, Edmonton, Alberta, Canada

$5{ }^{2}$ Department of Computer Science, University of Saskatchewan, Saskatoon, Saskatchewan, 6 Canada

7

8 Corresponding Author:

9 Alexander Doroshenko ${ }^{1}$

10 5-30 University Terrace, 8303-112 Street NW, Edmonton, Alberta T6G2T4, Canada 11

12 Email address: adoroshe@ualberta.ca

13

14

15

16

17

18

19

20

21

22

23

24

25

26

27

28

29 


\section{Abstract}

Background. Pertussis control remains a challenge due to recently observed effects of waning immunity to acellular vaccine and suboptimal vaccine coverage. Multiple outbreaks have been reported in different ages worldwide. For certain outbreaks, public health authorities can launch an outbreak response immunization campaign to control pertussis spread. We investigated effects of an outbreak response immunization targeting young adolescents in averting pertussis cases. Methods. We developed an agent-based model for pertussis transmission representing disease mechanism, waning immunity, vaccination schedule and pathogen transmission in a spatiallyexplicit 500,000-person contact network representing a typical Canadian Public Health district. Parameters were derived from literature and calibration. We used published cumulative incidence and dose-specific vaccine coverage to calibrate the model's epidemiological curves. We endogenized outbreak response by defining thresholds to trigger simulated immunization campaigns in the 10-14 age group offering $80 \%$ coverage. We ran paired simulations with and without outbreak response immunization and included those resulting in a single ORI within a 10-year span. We calculated the number of cases averted attributable to outbreak immunization campaign in all ages, in the 10-14 age group and in infants. The count of cases averted were tested using Mann-Whitney U test to determine statistical significance. Numbers needed to vaccinate during immunization campaign to prevent a single case in respective age groups were derived from the model. We varied adult vaccine coverage, waning immunity parameters, immunization campaign eligibility and tested stronger vaccination boosting effect in sensitivity analyses.

Results. 189 qualified paired-runs were analyzed. On average, ORI was triggered every 26 years. On a per-run basis, there were an average of 124, 243 and 429 pertussis cases averted across all age groups within 1,3 and 10 years of a campaign, respectively. During the same time periods, 53, 96, and 163 cases were averted in the 10-14 age group, and 6, 11, 20 in infants under 1 ( $<<0.001$, all groups). Numbers needed to vaccinate ranged from 49 to 221, from 130 to 519 and from 1031 to 4903 for all ages, the 10-14 age group and for infants, respectively. Most sensitivity analyses resulted in minimal impact on a number of cases averted.

Discussion. Our model generated 30 years of longitudinal data to evaluate effects of outbreak response immunization in a controlled study. Immunization campaign implemented as an outbreak response measure among adolescents may confer benefits across all ages accruing over 
61 a 10-year period. Our inference is dependent on having an outbreak of significant magnitude

62 affecting predominantly the selected age and achieving a comprehensive vaccine coverage

63 during the campaign. Economic evaluations and comparisons with other control measures can

64 add to conclusions generated by our work.

65

66

67

68

69

70

71

72

73

74

75

76

77

78

79

80

81

82

83

84 


\section{Introduction}

\subsection{Recent epidemiology of pertussis}

In recent years, pertussis control has re-emerged as a prominent public health challenge, with multiple outbreaks observed worldwide ${ }^{1}$, and with some jurisdictions reporting the highest numbers of cases seen in decades. ${ }^{2}$ In Canada, the last peak in pertussis activity was seen in the mid-1990s, after which incidence rates were gradually declining prior to a 2012 resurgence. This recent increase was driven by outbreaks in several provinces/territories. ${ }^{3}$ The national agespecific incidence rate remains highest among infants under 12 months of age, an age group also suffering the most hospitalizations and deaths. However, recently school-age children and younger adolescents have also borne a disproportionate burden, particularly during outbreaks. During the 2012 New Brunswick outbreak, the highest age-specific incidence rate fell in the 1014 age group, which was twice as high as the incidence rate among infants (1240 vs. 660 per 100,000, respectively). ${ }^{4}$ Such increases in incidence rates among older children were reported from several US states ${ }^{2,5}$, suggesting a bimodal age distribution of cases in some jurisdictions. During three most recent Minnesota outbreaks, the proportion of pertussis cases among children 7 to 18 years old exceeded $60 \%{ }^{6}$

The recent increase in pertussis activity is thought to be due to a combination of waning immunity from acellular pertussis vaccine and sub-optimal vaccine coverage. In the Ontario outbreak, cases were reported among unvaccinated individuals from a religious community and among vaccinated school-aged children. ${ }^{8}$ In New Brunswick, $67 \%$ of cases in the $10-14$ age group were up-to-date with their immunization. ${ }^{4}$ Several studies estimated the annual decline in protection after pertussis vaccination as ranging from $21 \%$ to $62 \% .{ }^{9,10,11}$ Vaccine-derived protection among individuals who were primed with the whole-cell pertussis vaccine is reported to be greater compared to individuals who received purely acellular formulations. ${ }^{12}$ Furthermore, natural disease confers even greater -- but not life-long -- protection. ${ }^{13}$ Genetic mutations in the Bordetella pertussis bacterium and better detection and diagnosis have been suggested as other explanations for this recent pertussis trend. ${ }^{14}$

Vaccination remains a cornerstone of public health measures to control pertussis. Improving immunization schedule adherence by raising awareness among public is the most commonly 
115 used intervention. The strategy of "cocooning" infants (vaccinating parents and other individuals

116 in close contact with infants) has been advocated, with mixed reviews. ${ }^{15,16}$ Immunizing pregnant

117 women in the third trimester of pregnancy to prevent pertussis disease in infants too young to

118 receive vaccination is recommended in the US. ${ }^{17}$ Modifications of the immunization schedule

119 (changing the age of vaccine administration or adding doses) have been discussed. ${ }^{18}$ Developing

120 new vaccines will offer the best long-term control strategy, however it is not likely to occur in

121 the short term. ${ }^{19}$

122 The ongoing occurrence of pertussis outbreaks presents a challenge to public health authorities

123 which may necessitate supplementary control measures. In Canada, immunization of pregnant

124 women is recommended only in outbreak situations. ${ }^{20}$ Early contact tracing and

125 chemoprophylaxis of contacts has been advanced as protective in control of school-based

126 outbreaks. ${ }^{21}$ Outbreak response immunization (ORI) has been employed if a particular group is

127 disproportionately affected and it is feasible to reach and vaccinate this group in a relatively

128 short period of time. ${ }^{4}$ ORI is supplementary immunization given over and above the routine

129 vaccination schedule, including to those who may be fully immunized or those who did not

130 receive their scheduled vaccines. Potential benefits of ORI could accrue both in the short-term

131 (terminating or limiting an ongoing outbreak) and long-term (preventing future outbreaks) and

132 may extend to age groups other than the age group for whom ORI was targeted. The relevance

133 of this intervention to all population (all other age groups) reflects the fact that interrupting

134 transmission among mostly affected group may also decelerate transmission to other age groups

135 with whom these individuals come in contact. Furthermore, it is very important to see whether

136 stopping outbreak in the ORI target group can have a material effect on a particularly vulnerable

137 population - babies under 1 year of age. ORI may also blunt natural boosting from circulating

138 sub-clinical infections. The cost of such immunization campaigns, including emergency response

139 infrastructure, cost of vaccines and their delivery is high, and often not included in routine

140 immunization programs budgets. Evaluation of such immunization campaigns is limited and the

141 need for pertussis outbreak response research has been advocated. ${ }^{22}$ The objective of our study

142 was to investigate the effect of outbreak response immunization (ORI) among adolescents as an

143 emergency public health intervention in light of a recent re-emergence of pertussis outbreaks. 


\subsection{Modeling approaches for pertussis}

146 Previous studies have used aggregate deterministic and stochastic models to understand pertussis

147 epidemiology and transmission and effects of vaccinations. In Grenfell and Anderson's

148 deterministic model, reduction of pertussis cases attributed to vaccination was estimated by

149 comparing pre-vaccine and vaccine eras. ${ }^{23}$ Hethcote's model, originally published in 1997,

150 introduced differential levels of immunity and infectiousness. ${ }^{24}$ Hethcote subsequently

151 contributed multiple adaptations of this model. ${ }^{25,26}$ Hethcote's models were adapted by other

152 authors to evaluate effects of delays in pertussis immunization, improving vaccine coverage ${ }^{27,28}$

153 and effectiveness of a routine adolescent booster. ${ }^{29}$ Wearing et al. used modeling to estimate

154 duration of pertussis immunity. ${ }^{30}$ Gabmhir et al. used US surveillance data to fit the model to

155 estimate epidemiological and vaccine-related parameters responsible for recent increases in

156 pertussis activity and demonstrated difference in duration of protection conferred by acellular

157 versus whole-cell vaccine. ${ }^{31}$ Sanstead et al. developed an agent-based model to characterize

158 pertussis outbreaks in Minnesota. ${ }^{6}$

\section{$159 \quad 1.3$ Rationale for ABM}

160

161

162

163

164

165

166

167

168

169

170

171

172

173

174

Impacts of interventions such as ORI cannot be summarized directly by collecting surveillance data because of the lack of controls (absent ORI intervention for the same outbreak). By contrast, such features and the complex interplay of waning immunity, network-mediated transmission, falling vaccination coverage, immunity boosting effects of exposure, and ORI and routine vaccination schedules make this investigation well-suited to agent-based simulation modeling. ${ }^{32}$ Such models can be used to systematically evaluate health outcomes during pertussis outbreaks in an otherwise identical context in the presence and absence of ORI. An agent-based modeling approach was selected here due to several characteristics of the system involved, including -- but not limited to -- the important role of individuals' connections, the spatially clustered character of outbreaks, the need for a finer-grained representation of both age and waning immunity, and the need for a longitudinal lens to understand the impact of individual vaccination compliance on vulnerability and to calibrate vaccination coverage data. An agent-based approach was further valuable for representing certain ORI intervention scenarios, particularly the restriction of administration of ORI to those with particular classes of vaccination histories. 


\section{Methods}

176 We developed an agent-based simulation model (ABM) and estimated the age-specific effects of

177 the pertussis ORI campaign in the 10-14 age group in simulated outbreaks in terms of the

178 number of cases averted over the short-, medium- and long-term (1, 3 and 10 years following

179 ORI implementation) among young adolescents (ORI target group), infants under 1 year and

180 individuals of all ages. This study was approved by the Health Research Ethics Board at the

181 University of Alberta, study ID Pro00050642.

182

183

184

185

186

187

188

189

190

191

192

193

194

195

196

197

198

199

200

201

202

203

204

\subsection{Model structure and agents characteristics}

The essential structure of the agent-base model is shown in figure 1A. Agents representing individual persons were associated with both fixed attributes and evolving states. Fixed attributes included a location (detailed below) and vaccination attitude, while evolving aspects of agent state included (continuous) age, count of vaccinations received and count of pertussis infections contracted. Statecharts were used to represent the natural history of infection, demographics and vaccination schedule.

The natural history of infection statechart drew its structure from the characterization in Hethcote's widely published and adapted compartmental model. ${ }^{24}$ As described in previous contributions (albeit at a compartmental rather than individual level), this representation includes 3 levels of severity of infection (I-full, I-mild, I-weak), and four levels of vaccination- (V1-V4) and naturally-induced (R1-R4) immunity and transitions between V, R and I states (with time and rate constants $\tau, \alpha$ and $\gamma$ ). Transitions were expressed by time-based and exposure-based formulations (e.g., 2 year increments to transition from higher to lower V states and 5 years to transition from higher to lower R states). In order to more realistically model the shapes of epidemiological curves over time to capture the outbreak dynamics that is critical to ORI triggering algorithms, we added incubation periods to the state charts, based on triangular distribution. While earlier adaptions of the Hethcote formulation typically assumed random mixing, in our model, individuals are importantly exposed to pathogens over a contact network (see below). In both cases, infection transmission is only possible to individuals who are susceptible (S state) or in the lower two levels of vaccine- and naturally- induced immunity. Vaccination and pathogen exposure boosts the level of both vaccine- and naturally induced immunity; waning of immunity decreases such immunity over time. While in an infected state, 
205 individuals in our model expose network connections, chosen with uniform probability, to 206 pathogen at an age-specific contact rate.

207 Demographic statecharts reflected a possible lifespan varying from 0 to 100 years and illustrated 208 individual mortality and (for female agents) fertility, with both being characterized using age209 specific hazard rates derived from Hethcote model. We used the 2004 Canadian population 210 pyramid to initialize the simulation (figure 1B).

211 The vaccination schedule statechart is modeled on North American vaccination regimes. It 212 characterizes possible vaccination episodes at ages 2, 4, 6 and 18 months, and 4-6 and 11-14 213 years (dose 6 is given at 11-12 years of age in the US and in grades 7 to 9 in Canada), with one 214 adult (18 years or older) vaccination also depicted. The range of ages when an adult dose is 215 administered in our model is between 18 and 35. This reflects the fact that parents of young 216 children may receive such a dose as a part of "cocooning" strategy. Alternatively, individuals 217 presenting to emergency departments with tetanus-prone wounds may also receive a pertussis218 containing vaccine. At each such age juncture, a person has a vaccine attitude- (acceptor, rejector 219 and hesitant) (supplementary tables 1A and 1B) and age- dictated probability of securing a 220 vaccination encounter; conditional on such an encounter, a vaccine is delivered. In our model, 221 we built a function to offer a catch-up vaccine if the previous dose is missed up to the minimal 222 age of the next dose (e.g., if $5^{\text {th }}$ dose is missed, an individual can have a chance to receive a 223 catch-up dose up to the age of 11 years). The probability of offering catch-up immunization is $22490 \%$, but this is conditional on an individual having a further encounter with a vaccine provider, 225 which in turn is dependent upon dose-specific vaccine coverage as well as contingent on 226 personal vaccine attitude (e.g., a vaccine rejecter will not have a catch-up vaccine offered). Each 227 occurrence of vaccination is associated with a fixed chance of vaccine failure (figure 1C). 


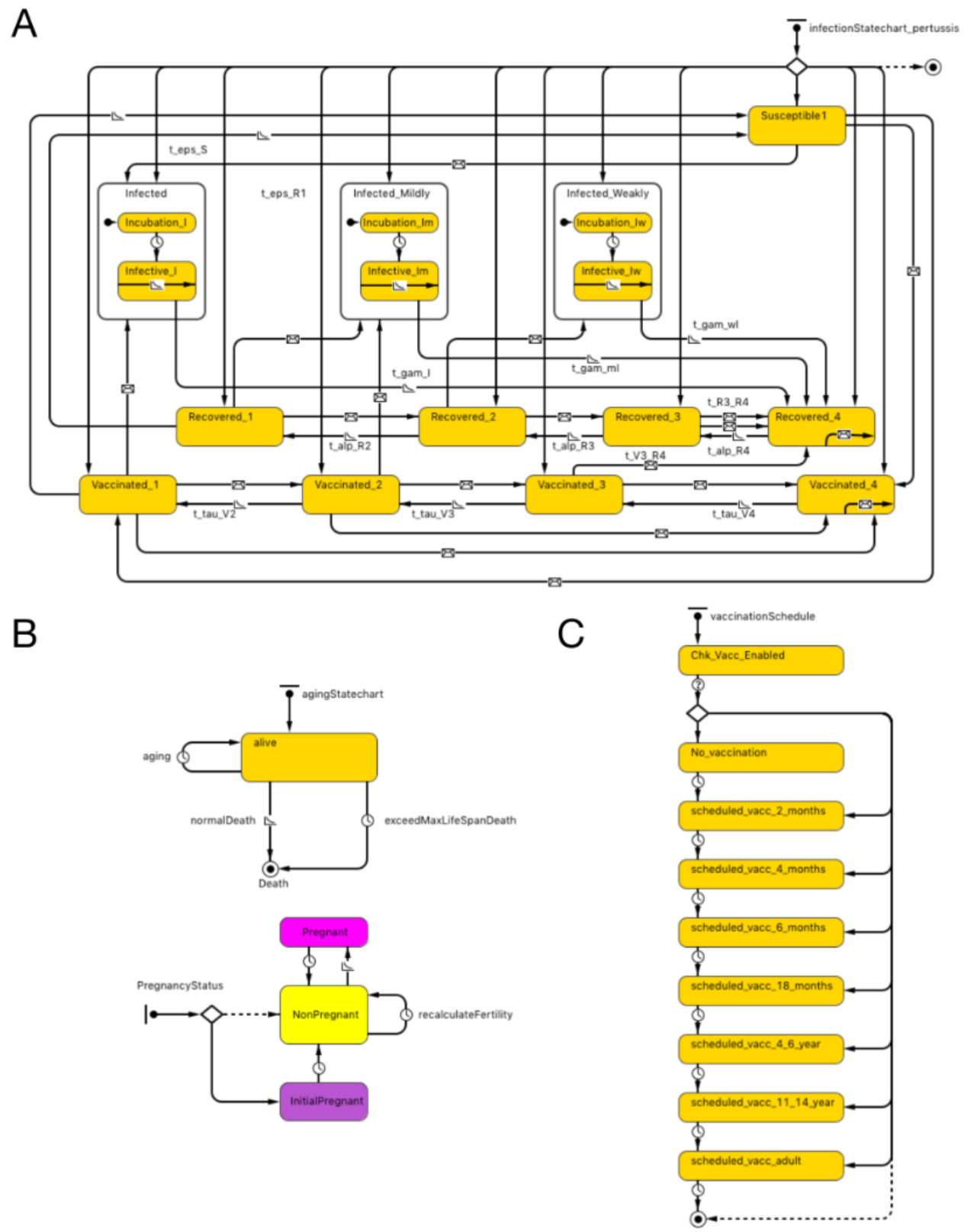

\section{Figure 1: Model structure.}

230 (A) Pertussis natural history statechart. (B) Demographic statechart. (C) Vaccination schedule 231 statechart.

\subsection{Network and spatial context}

233 To capture the spatially clustered nature of outbreaks, agents were distributed throughout a 234 stylized geographical area. The agent population was divided into a low density periphery 235 (constituting $29 \%$ of the population but $89 \%$ of the area), and a central region of 20 -fold higher 236 population density (holding the balance of the population and occupying the remaining area). 
237 Based on their spatial location, agents were placed in a quasi-static assortive network in which a

238 pair of agents was connected only if they lay within a specified distance threshold of each other.

239 The model used different distance thresholds governing whether a given pair of persons was

240 connected according to the age group of pair members. Specifically, while most pairs were

241 connected only if they were within a certain range of each other, if both members of the pair

242 were between 0 and 16 years old (inclusive), an 11-fold larger connection threshold was used

243 guided by calibration.

$244 \quad 2.3$ Parameterization

245 We configured our model using key parameters given in table 1. Disease mechanism parameters

246 pertaining to transitions between various $V, R$ and $I$ states were as described in the Hethcote

247 model. ${ }^{24}$ A primary vaccine failure probability described in the literature, ${ }^{9}$ and incubation

248 periods' range reflecting literature values ${ }^{33}$ were incorporated. Vaccine coverage was generated

249 by the model. To simulate the dynamics of vaccine coverage, we classified all individuals into

250 three groups: those who accept, reject and are hesitant to receive vaccination. For each of these

251 groups, we assigned vaccination probabilities. By adding network characteristic parameters and

252 an exogenous infection rate, we generated real-time epidemiological curves.

253

254

255

256 


\section{Table 1: Model's configuration and key parameters}

\begin{tabular}{|c|c|c|c|}
\hline Parameter Category & Parameter Name & Description & Value \\
\hline Demographics & Population size, (Persons) & $\begin{array}{l}\text { Population size at the model's' } \\
\text { initialization }\end{array}$ & 500000 \\
\hline \multirow{4}{*}{ Disease mechanism } & Incubation period, (Days) & $\begin{array}{l}\text { Incubation period corresponding to } \\
\text { different } I \text { states }\end{array}$ & $\begin{array}{l}\text { Follows triangular distribution }(\min =\{7,10,14\} \\
\text { mode }=\{10,14,21\}, \max =\{14,21,42\})\end{array}$ \\
\hline & $\begin{array}{l}\text { Mean waning time between } \mathrm{R} \text { states, } \\
\text { (Years) }\end{array}$ & \multirow{3}{*}{$\begin{array}{l}\text { Determine vaccine-derived and natural } \\
\text { disease-derived waning immunity }\end{array}$} & 5 \\
\hline & $\begin{array}{l}\text { Mean waning time between V states } \\
\text { (whole-cell vaccine), (Years) }\end{array}$ & & 2 \\
\hline & $\begin{array}{l}\text { Mean waning time between V states } \\
\text { (acellular vaccine), (Years) }\end{array}$ & & 2 \\
\hline Disease propagation & Exogenous infection Rate, (1/Day) & Represents imported infections & 5 \\
\hline \multirow{5}{*}{ Network characteristics } & Connection range, (Length) & \multirow{5}{*}{$\begin{array}{l}\text { Control mixing patterns and cumulative } \\
\text { incidence and shape of epidemiological } \\
\text { curve over time generated by the model }\end{array}$} & $\{$ Preferential $=55$, Normal $=5\}$ \\
\hline & Base contact rate, (1/Day) & & $\{$ Preferential $=20$, Ordinary $=3\}$ \\
\hline & Preferential mixing age, (Years) & & $\{$ from $=0$, to $=16\}$, years \\
\hline & $\begin{array}{l}\text { Base population density, } \\
\left(\text { Persons/Length }{ }^{2}\right)\end{array}$ & & 0.002 \\
\hline & Central-outer density ratio, (Unit) & & 20 \\
\hline
\end{tabular}


\begin{tabular}{|l|l}
\hline Vaccine coverage & Initial distribution of vaccination
\end{tabular} attitude in population, $\%$

Determine vaccine coverage generated by the model
$\{$ Vaccine Acceptor $(\mathrm{HA})=50$, Vaccine Hesitant $(\mathrm{VH})=40$, Vaccine Rejector $(\mathrm{VR})=10\}$ 
259

260

261

262

263

264

265

266

267

268

269

270

271

272

273

274

275

276

277

278

279

280

281

282

283

284

285

286

287

288

\subsubsection{Outbreaks and ORI triggers}

We developed an automated algorithm for triggering ORI (supplementary figures 1A-1C). The incidence rate of each month for the each age group was assigned a trichotomous $\mathrm{S}$ [suboutbreak] tag $[\mathrm{S}-, \mathrm{S}, \mathrm{S}+] . \mathrm{S}$ and $\mathrm{S}+$ states would require exceedance of the 60 -month moving average (excluding designated outbreaks) by 2 and 3 standard deviations, respectively and, additionally, exceedance of a specified monthly age-specific incidence rates (40 and 60 per 100,000 , respectively); the latter being derived by examining surveillance and outbreak reports 2,4,5,34 and further by calibration. An outbreak was defined as occurring if there were at least two consecutive months in the S state while ORI was triggered only in a setting of three consecutive months in $\mathrm{S}$ states or two consecutive months in $\mathrm{S}+$ states. We computed the rates of simulated outbreaks and ORI occurrence by dividing the number of outbreaks and ORI interventions within a specified age group across all realizations by the product of a total number of realizations in a given experiment and the 30 years in each run (i.e., model run-years). For this study, we only triggered ORIs in the 10-14 age group. Reciprocals of the outbreak and ORI rates represent the mean period between outbreaks within a given age group and time between ORIs, respectively. The latter was used to evaluate sensitivity of our model to triggering ORIs. ORI implementation was modeled as achieving $80 \%$ vaccination coverage for all individuals aged 10-14 at the time of ORI administration.

\subsection{Calibration and validation}

To better capture epidemiological trends of pertussis, we employed both quantitative and phenomenological approaches to validate and calibrate outputs from our model. More specifically, for the latter, we used the pattern-oriented modeling (POM) technique ${ }^{35,36,37}$ to optimize our model structure to ensure that generated epidemiological curves are realistic. We used this bottom-up strategy ${ }^{37}$ to model the highly complex system to improve our model robustness in a situation when interplay of multiple factors governing pertussis transmission, outbreak propagation and vaccination dynamic are less predictable and the understanding of the characteristics of the causal pathways involved remains incomplete. We extensively varied most of our network parameters (connection range, contact rate, exogenous infections rate) and studied resultant patterns by visual inspection of baseline endemic activity, age-specific peaks of outbreaks and intervals between outbreaks using a graphical-user interface. We identified 
289 obviously unrealistic outputs and gradually converged towards a well-fitting combination of 290 parameters.

291 Quantitatively, we adjusted model parameters to better match empirical cumulative incidence 292 and dose-specific vaccine coverage. We calibrated the model to bring the 30-year cumulative 293 incidence generated by the model in line with surveillance reports from two public health 294 jurisdictions of similar population size in Alberta (figure 2A and supplementary figure 2).

295 Comparability was defined as no more than 10\% deviation between model-generated average 296 cumulative incidence and that from two reference public health districts. Age-specific incidence 297 rates were checked during calibration as described above to ensure that age groups with the 298 highest burden of disease in our model were comparable to those of reference populations.

299 We validated waning immunity outputs with data derived from literature. We defined vaccine300 derived and natural infection-derived waning immunity in the context of ABM logic as transition 301 from the (protected) V3 (or R3) state to (unprotected) V2 (or R2) for each year. We generated a 302 model output for waning immunity and illustrated it for the 4-16 age group (figure 2B and 303 supplementary figures $3,4 \mathrm{~A}$ and $4 \mathrm{~B})$.

304 We calibrated vaccine coverage (as defined by receipt of all eligible doses) by varying 305 proportions of individuals by their vaccination attitudes and vaccination probabilities assigned to 306 their respective vaccination attitudes. The calibrated model was compared against vaccine 307 coverage statistics for doses 1 to 4 at age 2 published by Alberta Health ${ }^{38}$ in the same public 308 health jurisdictions used for cumulative incidence. Reference values for doses 5, 6 and 7 vaccine 309 coverage were obtained from other Canadian sources ${ }^{39}, 40$ given the reduced certainty for these 310 doses in Alberta (figure 2C). 


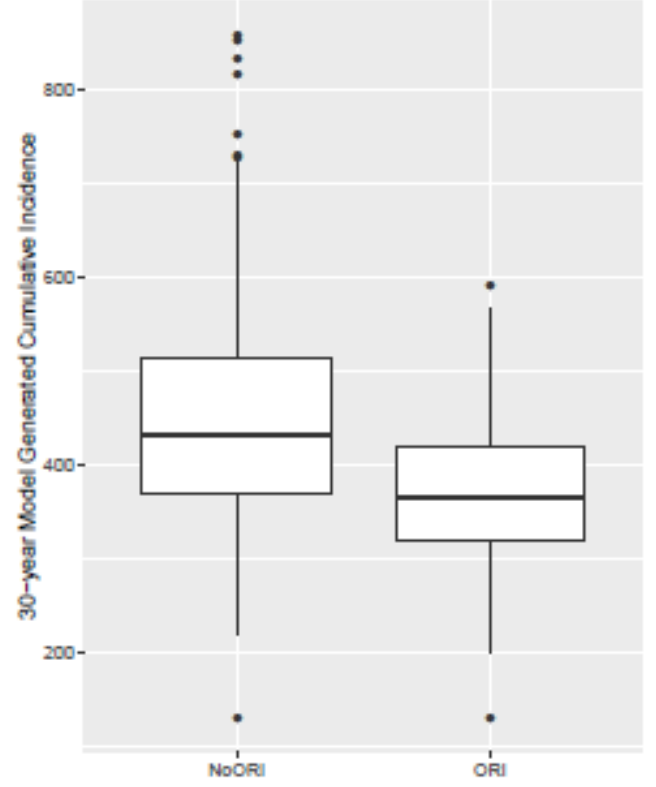

A
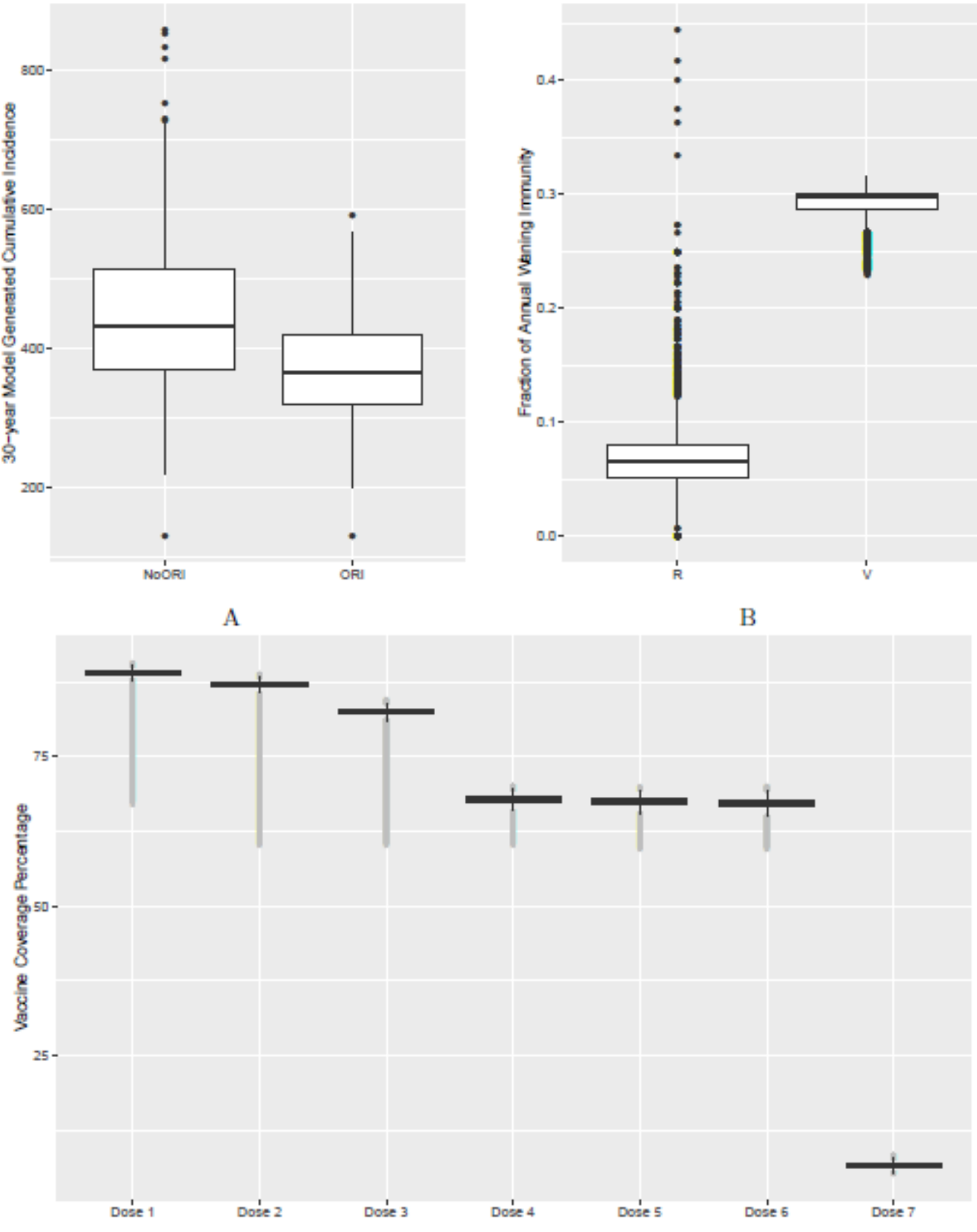

B

316 Figure 2: Model's output validation and calibration.

317 (A) 30-year model-generated cumulative incidence. (B) Vaccine- and natural disease-derived 318 waning immunity fractions. (C) Vaccine coverage by dose (doses 1 to 7). Model-generated 319 outputs depicted in (A), (B) and (C) are compared to 30-year cumulative incidence derived from 320 surveillance data from two Alberta jurisdictions, waning immunity values described in the 321 literature and dose-specific vaccine coverage derived from Canadian data sources respectively. 
322 To further test robustness of our model, we examined distribution of model-generated annual 323 incidence rates versus annual incidence rates distribution derived from empirical surveillance 324 data from two Alberta jurisdictions (figure 3). We tested differences between these two data 325 distributions by Mann-Whitney U-test $(\mathrm{p}$-value $=0.1068)$ and by Kolmogorov-Smirnov test $(\mathrm{p}$ 326 value $=0.1075$ ) with results indicating reasonable representation of simulated data versus 327 empirical data without evidence of overfitting.

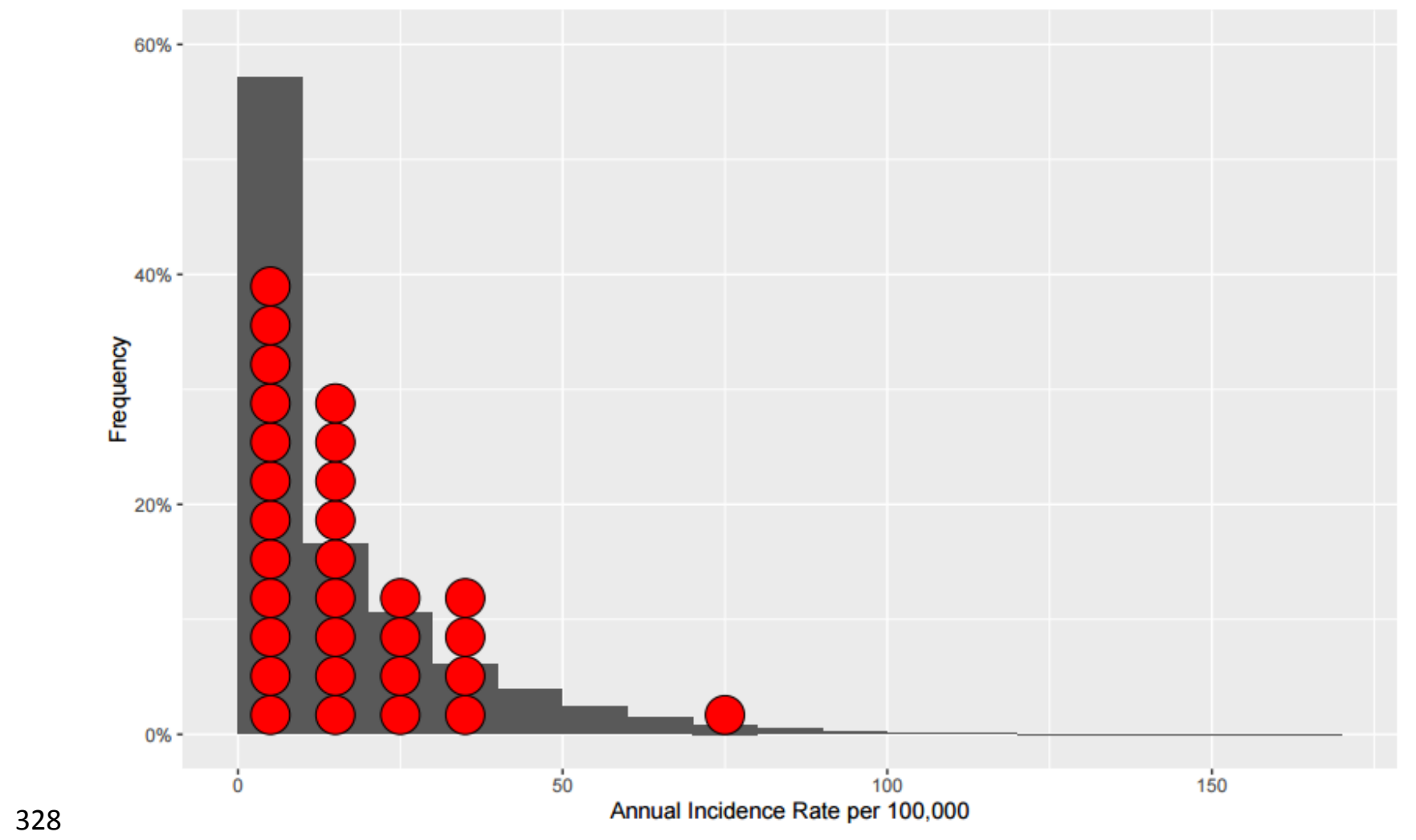

329 Figure 3: Distribution of the model-generated annual incidence rates in relation to empirical data. Bars represent frequencies corresponding to a particular annual incidence rate for the model-generated data. Red circles represent reference populations' (Alberta Central and South Zones) annual incidence rates. The total number of data points (annual incidence rates) based on which frequencies are computed for model-generated data is 10,200.

\subsection{Sensitivity Analyses}

As vaccine coverage for adult dose 7 may have the greatest uncertainty due to the lack of a fixed delivery age and underreporting of vaccination implemented as part of a cocooning strategy, we ran a sensitivity analysis increasing vaccine coverage for dose 7 by $20 \%$ (sensitivity analysis A). 
338 We further investigated the impact of waning immunity with a sensitivity analysis that reduced

339 annual waning immunity by increasing transition time between $\mathrm{V}$ states $(\tau)$ by $50 \%$ (from 2 to 3

340 years) among individuals born before 1997, representing the receipt of whole-cell vaccine

341 (sensitivity analysis B).

342 Additionally, we performed sensitivity analyses by reducing naturally-derived waning immunity 343 by doubling transition time between R states ( $\alpha$ ) from 5 to 10 years (sensitivity analysis C) and

344 by imposing eligibility restriction to receive ORI vaccination to only those who did not receive a 345 regular vaccine within last 6 months (sensitivity analysis D).

346 We also performed sensitivity analysis by including a scenario positing a more rapid boosting

347 effect from administration of vaccination (sensitivity analysis E). In this scenario, booster

348 immunization for an individual in lower V states would lead immediately to a V4 state (full

349 protection), without the multi-dose transition through V2 and V3 states as in the main

350 experiment. Finally, we conducted a multi-way sensitivity analysis (sensitivity analysis F) where

351 we considered the effects of simultaneous changes in $(\tau)$ and $(\alpha)$.

\section{$352 \quad 2.5$ Simulation setup and statistical analysis}

353 An open model population of initial size 500,000 was simulated in continuous time using

354 AnyLogic 7 software. We run multiple paired simulations (using identical random seeds) with

355 and without enabling the automated ORI module for 33 years. The first three years of simulation

356 were designated as a "burn-in period" and discarded, resulting in a 30-year period of

357 observations per simulation run. To yield meaningful results, statistical analysis was only

358 performed on "qualified" pairs of simulations, as judged by the following criteria:

359 i. At least one ORI was triggered within a simulation run;

360 ii. At least a 10-year post-ORI observation period was available;

361 iii. There was no second ORI triggered within a 10-year observation period;

362 Furthermore, qualified simulations meeting above criteria had to exhibit a cumulative incidence

363 rate comparable to two Alberta jurisdictions as described above.

364 We ran simulations on a high-power computer cluster at the University of Saskatchewan for 200

365 node-hours resulting in 334 pairs. Analyses were performed on data generated by the model. An 
366 illustration of differences between with-ORI and no-ORI case counts in a single paired

367 simulation run for all ages before and after a triggered ORI is shown in supplementary figure 5.

368 For each qualified pair, we calculated a number of cases averted within 1, 3 and 10 years after

369 ORI for three age groups: all ages, 10-14 (the ORI target age group) and infants under 1 year of

370 age (the most vulnerable group). The differences in the count of cases between the ORI and no-

371 ORI groups for a given qualified simulation pair were tested using the one-way Mann-Whitney

$372 \mathrm{U}$ test to determine statistical significance. We calculated a number needed to vaccinate during

373 ORI (NNV-ORI) to prevent a single case directly from the model by dividing a number of

374 vaccinations delivered during an ORI by a number of cases averted in a respective age-group.

375 This quantity will vary significantly in the context of different assumptions regarding the

376 population size and population immunity and therefore only applies to our model. Given multiple

377 simulations, we reported minimum and maximum NNV values.

\section{3. Results}

379189 qualified paired-runs met the inclusion criteria and were analyzed in the main experiment.

380 Characteristics and summary statistics of model-generated data are shown in table 2. In the main

381 experiment, the outbreak rate for all ages was 0.315 , indicating that outbreaks in all-ages group

382 were occurring approximately every 3 years in the model. The respective frequencies of outbreak

383 occurrence among adolescents 10-14 years of age and infants under 1 year of age were

384 approximately once in 7 and 13 years. Peak annual and monthly incidence rates for all ages

385 recorded in the main experiment across all realizations were 170 and 26 per 100,000,

386 respectively. Similarly, while the overall outbreak rates were lower in the 10-14 year category

387 and among infants when compared with those outbreak classifications considering all age

388 groups, peak annual and monthly incidence rates for children 10-14 years were 931 and 181 per

389100,000 , respectively, and for infants under 1 year of age, 725 and 207 per 100,000. On average,

390 an ORI campaign was triggered every 26 years. There were few variations in the rates of

391 outbreaks' occurrence and ORI triggering between the main experiment and all sensitivity

392 analysis scenarios other than sensitivity analysis involving more rapid boosting from vaccination

393 (sensitivity analysis E), which demonstrated significantly lower outbreak and ORI frequency.

394 The scenario in sensitivity analysis E was also the only situation where comparability criteria

395 with empirical data were not met. In the course of pattern-oriented modeling (POM) validation, 
396

397

398

399

we observed that reducing the exogenous infection rate resulted in a lower background incidence rate, punctuated by more pronounced outbreaks for a given cumulative incidence.

Table 2: Characteristics and summary statistics of the main experiment and sensitivity analyses

\begin{tabular}{|c|c|c|c|c|c|c|}
\hline & $\begin{array}{l}\text { Description of } \\
\text { parameter(s) alterations }\end{array}$ & $\begin{array}{l}\text { ORI rate in } \\
10-14 \text { age } \\
\text { group } \text { per } \\
\text { model run- } \\
\text { years }\end{array}$ & $\begin{array}{l}\text { Outbreak } \\
\text { rate in all } \\
\text { ages }{ }^{\text {p per }} \\
\text { model } \\
\text { run-years }\end{array}$ & $\begin{array}{l}\text { Outbreak } \\
\text { rate in } \\
\text { under } 19 \\
\text { per model } \\
\text { run-years }\end{array}$ & $\begin{array}{l}\text { Outbreak rate } \\
\text { in } 10-14 \text { age } \\
\text { group per } \\
\text { model run- } \\
\text { years }\end{array}$ & $\begin{array}{l}\text { Comparability } \\
\text { with } \\
\text { benchmark } \\
\text { cumulative } \\
\text { incidence }\end{array}$ \\
\hline $\begin{array}{l}\text { Main } \\
\text { experiment }\end{array}$ & Reference & 0.038 & 0.315 & 0.075 & 0.129 & Yes \\
\hline $\begin{array}{l}\text { Sensitivity } \\
\text { analysis A }\end{array}$ & $\begin{array}{l}\text { Increase vaccine coverage } \\
\text { for dose } 7 \text { by } 20 \%\end{array}$ & 0.037 & 0.315 & 0.080 & 0.127 & Yes \\
\hline $\begin{array}{l}\text { Sensitivity } \\
\text { analysis B }\end{array}$ & $\begin{array}{l}\text { Increase value of }(\tau) \text { to } 3 \\
\text { among those born after } \\
1997\end{array}$ & 0.039 & 0.323 & 0.075 & 0.127 & Yes \\
\hline $\begin{array}{l}\text { Sensitivity } \\
\text { analysis C }\end{array}$ & Increase value of $(\alpha)$ to 10 & 0.040 & 0.341 & 0.082 & 0.135 & Yes \\
\hline $\begin{array}{l}\text { Sensitivity } \\
\text { analysis D }\end{array}$ & $\begin{array}{l}\text { Restrict ORI eligibility to } \\
\text { those who did not receive } \\
\text { vaccine within last } 6 \\
\text { months }\end{array}$ & 0.036 & 0.324 & 0.078 & 0.129 & Yes \\
\hline $\begin{array}{l}\text { Sensitivity } \\
\text { analysis E }\end{array}$ & $\begin{array}{l}\text { Implement stronger } \\
\text { vaccine boosting effect }\end{array}$ & 0.005 & 0.047 & 0.013 & 0.021 & No \\
\hline $\begin{array}{l}\text { Sensitivity } \\
\text { analysis F }\end{array}$ & $\begin{array}{l}\text { Multi-way sensitivity } \\
\text { analysis B and C } \\
\text { combined }\end{array}$ & 0.025 & 0.325 & 0.083 & 0.129 & Yes \\
\hline
\end{tabular}

400

401

Notes:

$402 \S$ ORI rate is computed by dividing the number of triggered ORIs by the product of a total

403

404

405

406

407

408

409

number of simulation runs in a given experiment and 30 years in each run. Reciprocal of the ORI

rate represents mean time between occurrences of triggering ORIs; for example, the rate of 0.038

per model run-years in the main experiment indicates that ORI in the 10-14 age group was

triggered every 26 years in the model $(1 / 0.038=26.3)$.

I Outbreak rate is computed by dividing the number of outbreaks within a specified age group (or when judged with respect to all age groups) by the product of a total number of simulation runs in a given experiment and 30 years in each run. Reciprocal of the outbreak rate represents 
410 the mean period between outbreaks occurring within a given age group; for example, the rate of

4110.315 per model run-years in the main experiment indicates that outbreaks in all age groups were 412 occurring every 3 years in the model $(1 / 0.315=3.17)$.

413 \# Comparability with benchmark cumulative incidence was defined as model-generated 30-years 414 cumulative incidence rate falling within $10 \%$ of the average empirical cumulative incidence rate 415 derived from 15 years of observations in two jurisdictions in Alberta (15 years of observations 416 were up-scaled to derive 30-year cumulative incidence).

418 Vaccine-induced and natural disease-derived waning immunity rates in our model were 419 calculated to be $29 \%$ and $6.5 \%$ per year, respectively, in line with values reported from the 420 literature. ${ }^{9,13}$ Our model generated the following vaccine coverage for doses 1 to $7: 89 \%, 87 \%$, $42182 \%, 68 \%, 67 \%, 67 \%$ and $7 \%$, respectively (figure $2 \mathrm{C}$ and supplementary figures 3 and $4 \mathrm{~A}-4 \mathrm{~B}$ ).

422 In the main experiment, on a per-run basis, there were an average of 124, 243 and 429 pertussis 423 cases averted across all age groups within 1,3 and 10 years of a campaign, respectively. During 424 the same time periods, 53, 96, and 163 cases were averted in the 10-14 age group, and 6, 11, 20 425 in infants under 1 ( $\mathrm{p}<0.00001$ for all groups of comparisons of counts of cases in ORI versus no426 ORI simulations, one-way Mann Whitney U test). NNV-ORI ranged from 49 to 221, from 130 to 427519 and from 1031 to 4903 for all ages, the 10-14 age group and for infants, respectively (table 428 3). Boxplots for the number of cases averted for durations following ORI are depicted in figure 429 4, with each data point being associated with a particular realization. Over a 10-year period, there 430 431 was a gradual accrual of cases averted across all studied age groups; however, the accrual rate exhibited diminishing gains in years 5 through 10 . 
439 Table 3: Number of pertussis cases averted and numbers needed to vaccinate by time 440 periods after the outbreak-response immunization campaign and by age groups: modeling441 generated results

\begin{tabular}{|l|l|l|l|l|}
\hline Age groups & $\begin{array}{l}\text { Post-outbreak- } \\
\text { response immunization } \\
\text { period, years }\end{array}$ & $\begin{array}{l}\text { Average } \\
\text { number of } \\
\text { cases } \\
\text { averted* }\end{array}$ & $\begin{array}{l}\text { Minimum } \\
\text { number needed } \\
\text { to vaccinate }\end{array}$ & $\begin{array}{l}\text { Maximum } \\
\text { number needed } \\
\text { to vaccinate }\end{array}$ \\
\hline All ages & 1 & 124 & 171 & 221 \\
\hline All ages & 3 & 243 & 87 & 112 \\
\hline All ages & 10 & 429 & 49 & 64 \\
\hline Under 1 year & 1 & 6 & 3784 & 4903 \\
\hline Under 1 year & 3 & 11 & 1834 & 2377 \\
\hline Under 1 year & 10 & 20 & 1031 & 1336 \\
\hline $10-14$ years old & 1 & 53 & 400 & 519 \\
\hline $10-14$ years old & 3 & 96 & 220 & 285 \\
\hline $10-14$ years old & 10 & 163 & 130 & 168 \\
\hline
\end{tabular}

442

443 Notes:

$444 * \mathrm{p}<0.00001$ for all groups of comparisons of counts of cases in outbreak-response immunization 445 (ORI) versus no-ORI simulations, one-way Mann Whitney U test.

446 \$Number needed to vaccinate (NNV) was calculated directly from the model by dividing a

447 number of vaccinations delivered during the ORI by a number of cases averted in a respective 448 age group. NNV only applies to a current model and for a given population size. 
A

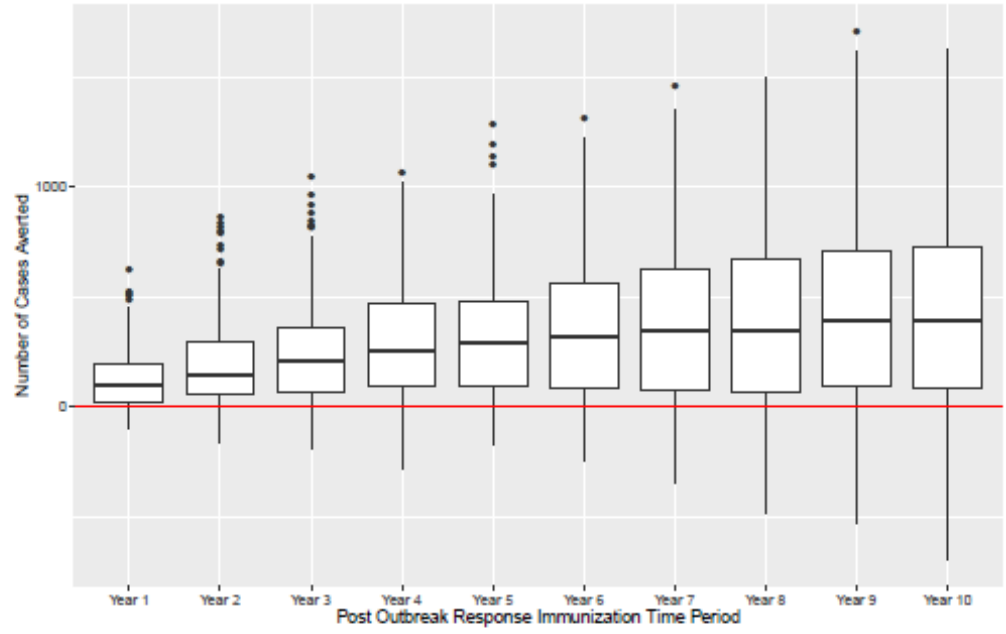

B

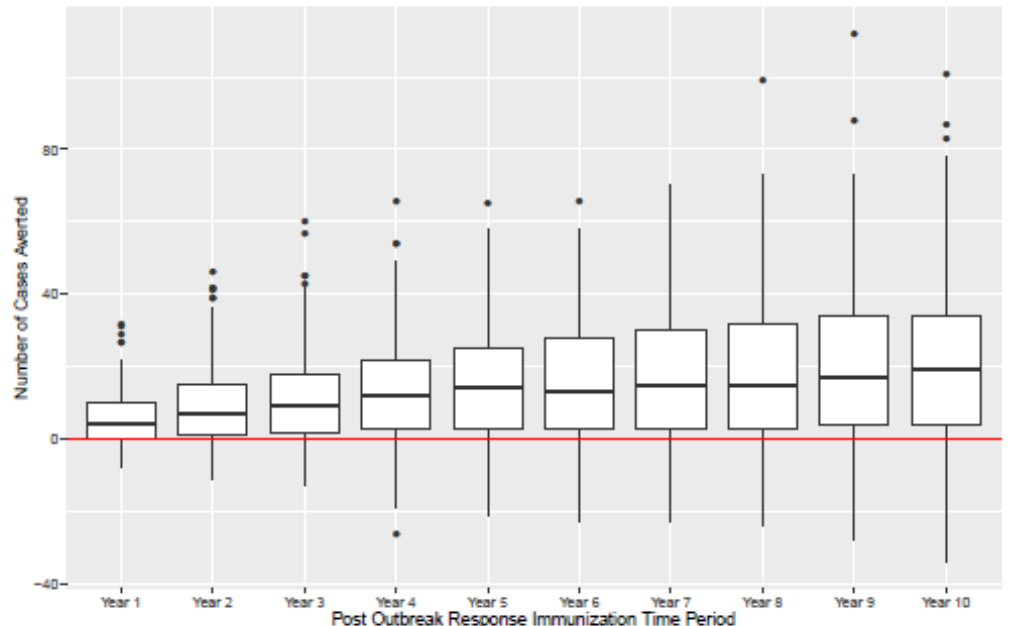

449

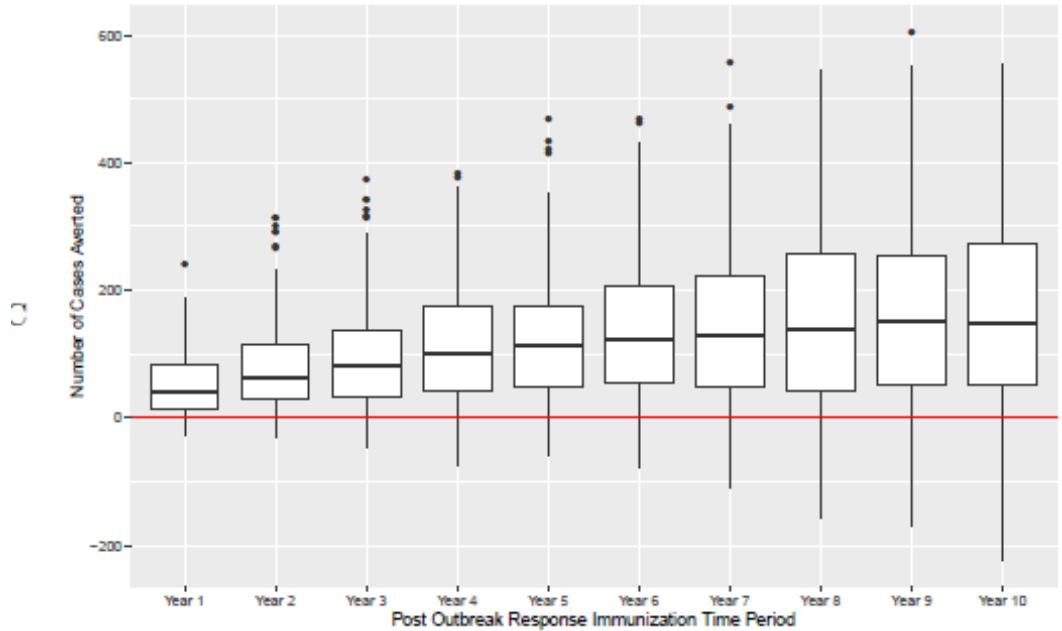

450 Figure 4: Number of pertussis cases averted over a 10-year period after implementation of 451 ORI campaign

452 (A) All ages group. (B) Infants under 1 year of age. (C) Adolescents 10-14 years of age 
453 Results of sensitivity analyses are summarized in table 4.

454 Table 4: Number of pertussis cases averted: summary of sensitivity analyses

\begin{tabular}{|c|c|c|c|c|c|c|c|c|}
\hline \multirow{2}{*}{$\begin{array}{l}\text { Age } \\
\text { group }\end{array}$} & \multirow{2}{*}{$\begin{array}{l}\text { Post- } \\
\text { ORI } \\
\text { period, } \\
\text { years }\end{array}$} & \multicolumn{7}{|c|}{ Number of cases averted ${ }^{\S}$ by experiment } \\
\hline & & $\begin{array}{l}\text { Main } \\
\text { experiment } \\
* \dagger\end{array}$ & $\begin{array}{l}\text { Sensitivity } \\
\text { analysis } \\
\mathrm{A}^{* \dagger}\end{array}$ & $\begin{array}{l}\text { Sensitivity } \\
\text { analysis } \\
\mathrm{B}^{* \dagger}\end{array}$ & $\begin{array}{l}\text { Sensitivity } \\
\text { analysis } \\
\mathrm{C}^{* \dagger}\end{array}$ & $\begin{array}{l}\text { Sensitivity } \\
\text { analysis } \\
\mathrm{D}^{* \dagger}\end{array}$ & $\begin{array}{l}\text { Sensitivity } \\
\text { analysis } \\
E^{*}\end{array}$ & $\begin{array}{l}\text { Sensitivity } \\
\text { analysis } \\
F^{* \dagger}\end{array}$ \\
\hline All ages & 1 & 124 & 119 & 112 & 148 & 108 & 41 & 130 \\
\hline All ages & 3 & 243 & 256 & 228 & 262 & 241 & 93 & 229 \\
\hline All ages & 10 & 429 & 410 & 409 & 422 & 429 & 148 & 378 \\
\hline Under 1 & 1 & 6 & 5 & 5 & 7 & 4 & $1 * *$ & 6 \\
\hline Under 1 & 3 & 11 & 12 & 10 & 13 & 10 & 4 & 10 \\
\hline Under 1 & 10 & 20 & 20 & 20 & 21 & 21 & 6 & 18 \\
\hline $\begin{array}{l}\text { Age } \\
10-14\end{array}$ & 1 & 53 & 51 & 48 & 61 & 47 & 19 & 54 \\
\hline $\begin{array}{l}\text { Age } \\
10-14\end{array}$ & 3 & 96 & 102 & 92 & 104 & 96 & 40 & 90 \\
\hline $\begin{array}{l}\text { Age } \\
10-14\end{array}$ & 10 & 163 & 156 & 156 & 161 & 162 & 60 & 143 \\
\hline
\end{tabular}

455

456 Notes:

$457 \S$ The number of cases averted was determined by subtracting count of pertussis cases in the

458 simulation ORI arm from the no-ORI arm within the same experiment for a given age group and

459 a given post-ORI period (count of cases expected to be lower in an ORI arm if cases are averted)

460 and expressed as an average on a per-run basis.

$461 * \mathrm{p}<0.00001$ for all groups of comparisons of counts of cases in outbreak-response immunization

462 (ORI) versus no-ORI simulations, one-way Mann Whitney U test (p-values apply to the entire

463 column).

464

$* * \mathrm{p}=0.12$

465 Prolonging the duration of vaccine-induced immunity among those who received whole-cell 466 vaccine had minimal impact on overall waning immunity and number of cases averted by ORI.

467 Prolonging the duration of natural disease-derived immunity resulted in such an annual waning 468 immunity decreasing to $2 \%$ but had minimal impact on the number of cases averted. Equally, 469 multi-way sensitivity analysis of combining both the effect of prolongation of whole-cell 470 vaccine-induced and natural disease-derived immunity had little impact on the number of cases

471 averted. Increasing vaccine coverage for dose 7 to $26 \%$ and restricting eligibility to receive 
472 vaccination during the ORI campaign to those who did not receive pertussis vaccine within 6

473 months had also minimal impact on cases averted relative to the baseline. However positing a

474 significantly stronger boosting effect from vaccination (bypassing V2 and V3 states in the state

475 chart in figure 1A) resulted in significantly fewer cases averted by ORI (148 cases averted in

476 sensitivity analysis E versus 429 in the main experiment for all-ages group over 10 years). Also,

477 the difference between ORI and no-ORI arms was not statistically significant for infants under 1

478 years of age at 1 year post ORI campaign $(p$-value $=0.12)$ in sensitivity analysis E. Interested

479 readers can refer to the supplemental material for additional results of sensitivity analyses

480 (supplementary tables 2A-2F and supplementary figures 6A-6F).

481 4. Discussion

482 Our ABM successfully generated 30 years of longitudinal data to evaluate the effects of 483 supplemental ORI in a controlled study. For this purpose, we expanded mechanisms widely 484 adopted from a previously published pertussis compartmental model by developing a spatially485 localized 500,000-person contact network representing a typical small-to-moderate size Canadian public health district, and also supplemented such elements with novel mechanisms to dynamically recognize outbreaks suitable for ORI, and trigger resulting immunization campaigns.

Modeling is used to enhance fundamental understanding of pertussis characteristics and transmission and to more pragmatically to evaluate impacts of interventions (e.g., adolescent or adult routine vaccination or cocooning strategy). While latter is often a subject of recent enquiries, our model, to our knowledge, is the first to represent and evaluate the effects of pertussis ORI. Such an ORI-specific evaluation is an important contribution to our understanding of outbreaks dynamics, as the force of infection of the sort of focused, large scale outbreak needed to motivate ORI may generate different transmission patterns which cannot be seen in the non-outbreak settings, and because ORI can re-shape both short- and long-term transmission dynamics either for the benefit or possibly to a detriment. The large scale outbreak itself may exhaust the pool of susceptibles and consequently yield a decrease in the number of cases in post-outbreak years, and lower incidence can lead to diminished natural boosting. For example, annual pertussis incidence rates were at historically low levels in 2 years following a large scale outbreak in New Brunswick in 2012 (187, 0.5 and 1.2 per 100,000 in 2012, 2013 and 2014, 
502 respectively) with a smaller outbreak reported in the third year. ${ }^{41,42}$ While this observation could

503 be due to the effect of the outbreak itself, the contribution of the ORI (which was implemented in 504 New Brunswick 2012 outbreak) is an important consideration.

505 We conclude that the effect of ORI is beneficial independently of the effect of the outbreak itself 506 and leads to a net number of cases averted in all age groups, particularly in the short and medium 507 term. While the objective of this model project focused on evaluation of the effects of ORI, the 508 model also supported a set of interesting secondary observations. We found that reducing the 509 exogenous infection rate resulted in a lower background incidence rate punctuated by more 510 pronounced outbreaks. This may suggest that jurisdictions with lower migration may be more 511 prone to larger scale but less frequent outbreaks, while jurisdiction with higher migration may 512 exhibit more frequent outbreaks with lower peak incidence. No significant changes to our 513 conclusions were observed from positing prolonged duration of natural disease-derived 514 immunity, increasing adult vaccine-coverage or restricting vaccination eligibility during ORI.

515 We observed no effect of altering the assumptions concerning waning immunity for those who 516 received whole-cell vaccine, which may be due to the fact that our model ran prospectively into 517 the future, with the number of individuals who had whole-cell vaccines progressively decreasing 518 over time. However, our findings in a sensitivity analysis that positing a stronger boosting effect 519 of vaccination implies a notably reduced burden of pertussis supports current thinking that 520 insufficient duration of immunity contributes to the recent resurgence of pertussis outbreaks.

521 One of the considerations in modeling/reproducing outbreaks is that, while historical surveillance 522 data plays an important role in defining whether an outbreak exist or not, the identification of an 523 outbreak is often judgement-based, with similar magnitude of pertussis incidence determined to 524 be an outbreak in a one jurisdiction, but not in others. We set outbreak and ORI thresholds in our 525 model high, effectively excluding instances of "borderline" outbreaks where ORI is unlikely to 526 ever be a consideration. As a result, ORI in our simulations was triggered once every 26 years 527 on average. This reflects the reality that ORI is not a commonplace intervention, particularly if 528 disease is endemic. In our model, we implemented ORIs only to adolescents 10-14 years of age, 529 reflecting recent outbreaks affecting this age group, who are largely fully immunized (and for 530 whom immunization schedule adherence was not protective) and their accessibility to school531 mediated campaigns; however, our model has the capability to test outbreak response in any age 
532 group. To ascertain whether ORI administered to young adolescents confer an indirect protection

533 to other age groups via interruption of transmission, we specifically examined the effects of ORI

534 administered to the adolescent age group on the number of cases averted among individuals of

535 all ages and among infants, as protecting infants is one the main priorities for public health

536 interventions. While we observed protective effect among adolescents and individuals of all

537 ages, our study revealed that a protective effect to infants is modest, as suggested by high NNV

538 generated by our model. These results are in the agreement with recent recommendations

539 concluding that a booster dose in adolescence or adulthood had minimal impact on infant

540 disease ${ }^{43}$; however, the latter recommendation was not specifically in the ORI context.

541 The main strength of our study is that we analysed longitudinal data generated by the model in a

542 manner of a controlled study, thus allowing us to independently evaluate and quantify the effects

543 of the ORI. As propagation of outbreaks depends on both intrinsic characteristics of individuals

544 as well as transmission-permitting connections, which exist between these agents, including both

545 characteristics in a single agent-based model allowed us to examine their interplay in outbreak

546 occurrence. Our model included age-structure to model pertussis vaccination and incorporated

547 vaccination attitudes into determination of vaccine coverage. Our model quantified both vaccine-

548 induced and natural disease-derived waning immunity. Furthermore, we calibrated and validated

549 the model by statistical comparison of the model-generated data and observed surveillance data

550 as well as by utilizing pattern-oriented modeling. Our model could be adapted, with varying

551 levels of ease, for different contexts and to investigate different types of research questions.

552 Adaptation to investigate similar ORI phenomena in other jurisdictions would involve a

553 circumscribed set of changes, including primarily changes to the vaccination statechart and

554 associated probabilities (to represent local vaccination regimes), probabilities associated with

555 vaccine attitude (reflecting differences in local attitudes towards vaccination), population sizes

556 and population density, and potentially age-specific mixing assumptions. With a greater degree

557 of modifications, and contingent on retaining current disease transmission logic, our model

558 would also permit to investigate effects of other public health interventions ranging from altering

559 vaccination schedules, evaluating effects of passive messaging to adhere to immunization

560 schedules and adding vaccine doses in adults. 
562 Our study has several limitations. We used disease mechanism parameters initially outlined in

563 the Hethcote model. While conducting several sensitivity analyses involving key parameters, our 564 experiments with different disease transmission logic were limited to enhancing boosting effect; 565 a broader set of altered assumptions in this area may or may not yield different results for our 566 research question. Recent study suggests that non-human primates vaccinated with acellular 567 pertussis vaccine were protected from severe symptoms, but not infection, and readily 568 transmitted Bordetella pertussis to contacts. ${ }^{44}$ In recent review of pertussis models Campbell et 569 al. identified incomplete understanding relating infection and disease and lack of supporting data 570 to derive parameters as common limitations of proposed pertussis models. ${ }^{45}$ While our

571 calibration process helped ensure that our model output is realistic, we did not test variations in 572 every single parameter given the multi-faceted nature of our model. Furthermore until further 573 knowledge emerges to narrow down or alter parameters value, using the classic model structures 574 on which our model is based would appear appropriate. We did not aim to examine and compare 575 public health strategies other than ORI, and the need to pursue such research is strong. Economic 576 evaluations can offer valuable additions to conclusions generated by our work.

577

\section{5. Conclusions}

579 We developed an agent-based model to investigate effects of outbreak response immunization 580 campaigns targeting young adolescents in averting pertussis cases. We concluded that such an 581 immunization campaign confers benefits across all age groups accruing over a 10-year period.

582 Our inference is dependent on having an outbreak of significant magnitude affecting

583 predominantly the selected age and achieving a comprehensive age-specific coverage rate during 584 the campaign. Our results demonstrated that while outbreak response may yield modest benefits 585 for protecting infants, additional strategies to protect this vulnerable group are needed. Our 586 experience indicates that ABM offers a promising methodology to evaluate other public health 587 interventions used in pertussis control. We also identify the strong need for further research into 588 application of modeling to further our understanding of pertussis epidemiology. 


\section{6. Acknowledgements}

593 Authors acknowledge contributions of public health departments in Alberta and New Brunswick 594 in obtaining surveillance data.

595

\section{7. References}

597 1. Tan T, Dalby T, Forsyth K. Pertussis across the globe: recent epidemiologic trends from 2010 598 to 2013. Pediatr Infect Dis J. 2015; 34(9):e222-32.

599 2. California Department of Public Health. Pertussis report. February 2015. Available at

600 http://www.cdph.ca.gov/programs/immunize/Documents/PertussisReport2-12-2015.pdf.

601 Accessed December 26, 2015.

602 3. Smith T, Rotondo J, Desai S, Deehan H. Pertussis surveillance in Canada: trends to 2012.

603 Canada Communicable Disease Report. 2014. Available at http://www.phac-

604 aspc.gc.ca/publicat/ccdr-rmtc/14vol40/dr-rm40-03/dr-rm40-03-per-eng.php. Accessed December $60526,2015$.

606 4. Office of the Chief Medical Officer of Health. New Brunswick, Canada. New Brunswick

607 pertussis outbreak investigation report. 2014. Available at

608 http://www2.gnb.ca/content/dam/gnb/Departments/h-

609 s/pdf/en/CDC/HealthProfessionals/PertussisReport.pdf. Accessed December 26, 2015.

610 5. Wisconsin Department of Health Services. 2012 Annual pertussis surveillance summary.

611 Available at https://www.dhs.wisconsin.gov/immunization/2012asrpertussis.pdf. Accessed

612 December 26, 2015.Oregon reference adolescent peak

613 6. Sanstead E, Kenyon C, Rowley S, Enns E, Miller C, Ehresmann K, Kulasingam S.

614 Understanding Trends in Pertussis Incidence: An Agent-Based Model Approach. Am J Public

615 Health. 2015; 105(9):e42-7

616 7. Public Health Agency of Canada. Canadian immunization guide. Pertussis vaccine. 2014.

617 Available at http://www.phac-aspc.gc.ca/publicat/cig-gci/p04-pert-coqu-eng.php\#a4. Accessed

618 December 26, 2015.

619 8. Deeks SL, Lim GH, Walton R, Fediurek J, Lam F, Walker C, Walters J, Crowcroft NS.

620 Prolonged pertussis outbreak in Ontario originating in an under-immunized religious community.

621 Canada Communicable Disease Report. 2014. Available at http://www.phac- 
622 aspc.gc.ca/publicat/ccdr-rmtc/14vol40/dr-rm40-03/dr-rm40-03-ont-eng.php. Accessed December $62326,2015$.

624 9. McGirr A, Fisman DN. Duration of pertussis immunity after DTaP immunization: a meta625 analysis. Pediatrics. 2015; 135(2):331-43.

626 10. Klein NP, Bartlett J, Rowhani-Rahbar A, Fireman B, Baxter R.

627 Waning protection after fifth dose of acellular pertussis vaccine in children. N Engl J Med. 2012;

$628 \quad 367(11): 1012-9$

629 11. Tartof SY, Lewis M, Kenyon C, White K, Osborn A, Liko J, Zell E, Martin S, Messonnier 630 NE, Clark TA, Skoff TH. Waning immunity to pertussis following 5 doses of DTaP.

631 Pediatrics. 2013; 131(4):e1047-52

632 12. Sheridan SL, Frith K, Snelling TL, Grimwood K, McIntyre PB, Lambert SB.

633 Waning vaccine immunity in teenagers primed with whole cell and acellular pertussis vaccine:

634 recent epidemiology. Expert Rev Vaccines. 2014; 13(9):1081-106

635 13. Wendelboe AM, Van Rie A, Salmaso S, Englund JA. Duration of immunity against pertussis 636 after natural infection or vaccination. Pediatr Infect Dis J. 2005; 24(5 Suppl):S58-61.

637 14. Jackson DW, Rohani P. Perplexities of pertussis: recent global epidemiological trends and 638 their potential causes. Epidemiol Infect. 2014; 142(4):672-84.

639 15. Rosenblum E, McBane S, Wang W, Sawyer M. Protecting newborns by immunizing family 640 members in a hospital-based vaccine clinic: a successful Tdap cocooning program during the 6412010 California pertussis epidemic. Public Health Rep. 2014; 129(3):245-51.

642 16. Healy CM, Rench MA, Wootton SH, Castagnini LA. Evaluation of the impact of 643 a pertussis cocooning program on infant pertussis infection. Pediatr Infect Dis J. 2015; 34(1):226446 
645 17. Centers for Disease Control and Prevention. Advisory Committee on Immunization

646 Practices. Updated Recommendations for Use of Tetanus Toxoid, Reduced Diphtheria Toxoid, 647 and Acellular Pertussis Vaccine (Tdap) in Pregnant Women. MMWR Morb Mortal Wkly Rep. $648 \quad 2013 ; 62(07) ; 131-135$.

649 18. Libster R, Edwards KM. Re-emergence of pertussis: what are the solutions? Expert Rev 650 Vaccines. 2012; 11(11):1331-46

651 19. Meade BD, Plotkin SA, Locht C. Possible options for new pertussis vaccines. J Infect 652 Dis. 2014; 209 Suppl 1:S24-7

653 20. Public Health Agency of Canada. National Advisory Committee on Immunization. An 654 Advisory Statement. 2014. Update on pertussis vaccination in pregnancy. Available at 655 http://www.phac-aspc.gc.ca/naci-ccni/acs-dcc/2014/pvip-vcpg 0214-eng.php. Accesses 656 December 26, 2015.

657 21. Miguez Santiyan A, Ferrer Estrems R, Chover Lara JL, Alberola Enguidanos J, Nogueira 658 Coito JM, Salazar Cifre A. Early intervention in pertussis outbreak with high attack rate in 659 cohort of adolescents with complete acellular pertussis vaccination in Valencia, Spain, April to 660 May 2015. Euro Surveill. 2015; 20(27).

661 22. World Health Organization. Pertussis working group report. 2019. Available at 662 www.who.int/immunization/sage/pertussis SAGE oct09.pdf. Accessed December 26, 2015. 663 23. Grenfell RT, Anderson RM. Pertussis in England and Wales: an investigation of transmission 664 dynamics and control by mass vaccination. Proc R Soc Lond B Biol Sci. 1989; 236:213-52.

665 24. Hethcote HW. An age-structured model for pertussis transmission. Math Biosci. 1997; 666 145(2):89-136.

667 25. Hethcote HW, Horby P, McIntyre P. Using computer simulations to compare pertussis 668 vaccination strategies in Australia. Vaccine. 2004; 22:2181-91.

669 26. Van Rie A, Hethcote HW. Adolescent and adult pertussis vaccination: computer simulation 670 of five new strategies. Vaccine. 2004; 22:3154-65. 
671 27. Pesco P, Bergero P, Fabricius G, Hozbor D. Mathematical modeling of

672 delayed pertussis vaccination in infants. Vaccine. 2015; 33(41):5475-80

673 28. Pesco P, Bergero P, Fabricius G, Hozbor D. Assessment of pertussis vaccination strategies

674 using a mathematical model of disease transmission. Arch Argent Pediatr. 2013; 111(5):377-83

675 29. Fabricius G, Bergero PE, Ormazabal ME, Maltz AL, Hozbor DF.

676 Modelling pertussis transmission to evaluate the effectiveness of an adolescent booster in

677 Argentina. Epidemiol Infect. 2013; 141(4):718-34

678 30. Wearing HJ, Rohani P. Estimating the duration of pertussis immunity using epidemiological

679 signatures. PLoS Pathog. 2009; 5:e1000647

680 31. Gambhir M, Clark TA, Cauchemez S, Tartof SY, Swerdlow DL, Ferguson NM. A change in 681 vaccine efficacy and duration of protection explains recent rises in pertussis incidence in the 682 United States. PLoS Comput Biol. 2015; 11(4):e1004138.

683 32. Mabry PL, Marcus SE, Clark PI, Lieschow SJ, Mendez D. Systems Science: A Revolution in 684 Public Health Policy Research. Am J Public Health. 2010; 100(7): 1161-1163.

685 33. Centers for Disease Control and Prevention. Pertussis. Epidemiology and prevention of 686 vaccine-preventable diseases. The Pink Book: course textbook - 13th edition. 2015. Available at 687 http://www.cdc.gov/vaccines/pubs/pinkbook/pert.html. Accessed December 26, 2015.

688 34. Minnesota Department of Health. Pertussis 2012. Available at

689 http://www.health.state.mn.us/divs/idepc/newsletters/dcn/sum12/pertussis.html. Accessed 690 December 26, 2015.

691 35. Grimm V, Revilla E, Berger U, Jeltsch F, Mooij WM, Railsback SF, Thulke HH, Weiner J, 692 Wiegand T, DeAngelis DL. Pattern-oriented modeling of agent-based complex systems: lessons 693 from ecology. Science. 2005: 310(5750): 987-991.

694 36. Topping CJ, Hoye TT, Olesen CR. Opening the black box - Development, testing and 695 documentation of a mechanistically rich agent-based model. Ecological Modelling. 2010; $696 \quad 221: 245-255$

697 37. Railsback SF, Grimm V. Agent-Based and Individual-Based Modeling: A Practical

698 Introduction. Princeton: Princeton University Press, 2012

699 38. Alberta Health. Interactive health data application. Available at

700 http://www.ahw.gov.ab.ca/IHDA Retrieval/. Accessed December 26, 2015. 
701 39. Public Health Agency of Canada. Vaccine coverage amongst adult Canadians: Results from 702 the 2012 adult National Immunization Coverage (aNIC) survey. Available at http://www.phac703 aspc.gc.ca/im/nics-enva/vcac-cvac-eng.php. Accessed December 26, 2015.

704 40. Public Health Ontario. Immunization coverage report for school pupils. 2014. Available at 705 https://www.publichealthontario.ca/en/eRepository/Immunization_coverage report 2012-13.pdf. 706 Accessed December 26, 2015.

707 41. Office of the Chief Medical Officer of Health. New Brunswick, Canada. New Brunswick 708 communicable disease annual report. 2013. Available at

709 http://www2.gnb.ca/content/dam/gnb/Departments/h-

710 s/pdf/en/CDC/HealthProfessionals/Annual_Report_CDC_Branch_2013.pdf. Accessed May 15, 7112016.

712 42. Office of the Chief Medical Officer of Health. New Brunswick, Canada. New Brunswick 713 communicable disease annual report. 2014. Available at

714 http://www2.gnb.ca/content/dam/gnb/Departments/h-

715 s/pdf/en/CDC/HealthProfessionals/Annual_Report_CDC_Branch_2014.pdf. Accessed May 15, 7162016

717 43. World Health Organization. SAGE Pertussis working group background paper. 2014.

718 Available at

719 http://www.who.int/immunization/sage/meetings/2014/april/1_Pertussis background_FINAL4 720 web.pdf. Accessed December 26, 2015.

721 44. Warfel JM, Zimmerman LI, Merkel TJ. Acellular pertussis vaccine protect against disease

722 but fail to prevent infection and transmission in a nonhuman primate model. Proc Natl Acad Sci $723 U S$ A. 2014; 111(2):787-92.

724 45. Campbell PT, McCaw JM, McVernon J. Pertussis models to inform vaccine policy. Hum 725 Vaccin Immunother. 2015; 11(3):669-78. 